\title{
Comparison of the Effectiveness of Oral Care with Chlorhexidine and Povidone iodine on the Incidence of Ventilator Associated Pneumonia in ICU - A prospective randomized controlled study.
}

\author{
Dr. Prakash Gupta ${ }^{1}$, Dr. Priyamvada Gupta ${ }^{2}$, Dr. D. D.Jethava ${ }^{3}$, \\ Dr. (Mrs) Durga Jethava ${ }^{4}$ \\ ${ }^{1,2,3,4}$ Department of Anesthesiology, Critical care and Pain management, Mahatma Gandhi Medical College, \\ Mahatma Gandhi University of Medical Science and Technology, India
}

\begin{abstract}
:
Background: Mechanical ventilation is often required in 10-20\% critically ill patients, may be because of respiratory or any other indication. There is always a risk of developing pneumonia in such patients. Any pneumonia developing after 48 hours of commencing mechanical ventilation in a patient with previously non infected lungs is labeled as ventilator associated pneumonia (VAP).It occurs in 9-27\% of patients on mechanical ventillation. This study was aimed at comparing the effectiveness of oral care with povidone iodine and chlorhexidine in preventing VAP.

Materials and methods: This prospective randomized study included 70 patients in critical care unit requiring mechanical ventilation for more than 72 hrs After obtaining permission from hospital ethical committee and written informed consent from the patients, they were randomly allocated in two groups of 35 each: group I and group II to receive oral care with povidone iodine and chlorhexidine respectively. Development of VAP was recorded on basis of clinical findings, $x$-ray and tracheal culture.

Results \& Conclusions: In group receiving oral care with povidone iodine, $6(11.4 \%)$ patients developed VAP as compared to chlorhexidine group only $4(17.1 \%)$ patients developed VAP but it did not reach statistical significance. Thus oral care with chlorhexidine is superior in prevention of VAP as compared with povidone iodine mouth wash but more studies need to be done, with larger sample size.
\end{abstract}

Keywords: Chlorhexidine, mouthwash, mechanical ventilation, povidone iodine, VAP (Ventilator Associated Pneumonia)

\section{Introduction}

Mechanical ventilation is often required in 10-20\% critically ill patients, may be because of respiratory or any other indication [1]. There is always a risk of developing pneumonia in such patients. Any pneumonia developing after 48 hours of commencing mechanical ventilation in a patient with previously non infected lungs is labeled as ventilator associated pneumonia (VAP)[2,3].VAP occurs in 9-27\% of all intubated patients[4,5].Intensive care units (ICUs) are the hot zones of nosocomial infections and antibiotic resistance. Many patients experience immune paralysis which is induced by an acute and severe disease, many have underlying immunodeficiency and/or significant co-morbidity and most of them need invasive devices. As a result intensive care unit patients on mechanical ventilation are extremely prone to infection. The most frequently occurring ICU acquired infection is ventilator associate pneumonia (VAP) [6].

Aspiration of oropharyngeal secretions into the bronchial tree is a major factor in the development of VAP. Besides this, poor oral hygiene can lead to bacterial colonization of the oropharynx. There is a strong correlation between the bacteria colonizing the oropharynx and those causing VAP in mechanically ventilated patients. Based on this hypothesis, we conducted this study comparing the effectiveness of oral care with two disinfectant solutions namely povidone iodine and chlorhexidine, in preventing VAP.

\section{Methodology}

It was a tertiary care hospital based prospective randomized study. Study included 70 mechanically ventilated ill patients in critical care unit during the study period. After obtaining permission from hospital ethical committee and written informed consent from the patients, they were randomly allocated in two groups of 35 each. Group I and Group II received oral care with povidone iodine and chlorhexidine respectively. Development of VAP in patients was recorded on the basis of clinical findings, $\mathrm{x}$-ray and tracheal culture.

Patients were randomized on the basis of computer generated table of random numbers. Inclusion criteria were: patients of age 18 to $60 \mathrm{yrs}$, requiring mechanical ventilation for more than $72 \mathrm{hrs}$, with normal hemodynamics and ABG.Exclusion criteria included patients with Acute Respiratory Distress Syndrome, Chronic Obstructive Lung Disease and active chest infection; patients already received mechanical ventilation 
for more than 24 hours;patients who have aspirated/with chest $\mathrm{x}$-rays already showing infiltrates.Clinical parameters were of hourly recordings of heart rate , temperature and blood pressure.

Investigations included daily TLC and DLC; chest $\mathrm{X}$ ray and endotracheal cultures on alternate days. Diagnostic criteria for labelling VAP included temperature $>38^{\circ} \mathrm{C}$ and $<36^{\circ} \mathrm{C}$, blood counts $>12000$ cells $/ \mathrm{mm} 3$ or $<4000$ cells $/ \mathrm{mm} 3$ with positive X-ray finding, positive culture of endotracheal aspirate $>10,00000 \mathrm{CFU} / \mathrm{M}$ or equivalent semi quantitive results.

Statistical analysis was done with IBM SPSS 20 statistical software and analyzed for various confounding factors via students $\mathrm{t}$ - test. An incidence of VAP was compared using chi square test and $\mathrm{p}$ value was calculated. $\mathrm{p}$ value $<0.05$ was considered statistically significant.

\section{Results}

Both the study groups were statistically identical (TABLE 1). Temperature variation was seen with 7 patients belonging to group I as compared to 4 patients in group II (Fig1)as was with tracheal culture (Fig2) $(\mathrm{p}=0.6)$ but the total leucocyte counts variation in group I was positive in 6 patients as compared to 4 patients in group II(Fig3)(p=0.7). X ray finding was also in accordance with the finding of total leucocyte count (Fig4). Although the patient developing VAP in group I was 6(11.4\%) as compared to 4 in group II (17.1\%) was not statistically significant ( $\mathrm{p} 0.61$ ).

\section{Discussion}

VAP is a common, morbid ICU complication of ventilated patients. Diagnosis of VAP is very challenging with high inter-observer variability, regularly audit prevention practices. The risk factors for VAP are underlying pulmonary disease, sepsis, acute respiratory distress syndrome, major surgery, multiple organ failure, head injury, witnessed aspiration, enteral nutrition, G I prophylaxis. Key in the pathogenesis of VAP is colonization of the upper respiratory tract (oropharynx and trachea) with potentially pathogenic microorganisms, such as Enterobatericeae, Staphylococcus aureus, and Pseudomonas aeruginosa.

The risk of VAP is highest early in the course of hospital stay, and is estimated to be $3 \%$ per day during the first 5 days of ventilation; $2 \%$ per day during days 5- 10 of ventilation; and $1 \%$ per day thereafter [7]. Crude mortality rates in patients with VAP range from $24-50 \%$, increasing to $76 \%$ if infection is caused by multiresistant organisms. Patients who develop VAP are twice as likely to die as those without VAP. It can be prevented by using VAP bundle of care along with other complication which results in increase morbidity and expenses of hospital. Because of its association with unwanted clinical outcomes, preventive measures have been studied extensively in the past 25 years. In a 1-day point-prevalence survey in 1417 ICUs worldwide in 2007 , the prevalence of respiratory tract infection was $64 \%$ among all patients infected [8]. Hospital-acquired pneumonia (HAP), ventilator-associated pneumonia (VAP), and healthcare-associated pneumonia (HCAP) remain important causes of morbidity and mortality despite advances in antimicrobial therapy, better supportive care modalities, and the use of a wide-range of preventive measures. Aspiration of oropharyngeal secretions into the bronchial tree is a major factor in the development of VAP [9]. Gastric colonization by potentially pathogenic organisms increases with lower gastric acidity [10]. Medications that alter the gastric $\mathrm{pH}$ may increase the number of organisms present and so increase the risk for VAP. Poor oral hygiene in mechanically ventilated patients can lead to bacterial colonization of the oropharynx. There is a strong correlation between the bacteria colonizing the oropharynx and those causing VAP [11].Early onset of VAP occurs in first 4 days of hospitalization and more likely to be caused by Moraxella catarrhalis, H. influenzae, or S. pneumonia. Late onset of VAP occurs 5 or more days into hospitalization and often caused by Gram-negative bacilli, or S. aureus (including methicillin resistant/MRSA), yeasts, fungi, legionellae and Pneumocystis carinii.

\section{Conclusion}

Oral care with chlorhexidine appears superior as compared to povidone iodine in the prevention of ventilator associated pneumonia but it did not reach statistical significance. Further studies are required with larger sample size and studying biochemical markers. Thus it may be one of the important components of VAP bundle of care along with other measures.

Acknowledgement: None

Support: Nil

Conflict of Interest: None 
VI. Figures and Tables

Table1. Demographic Data

\begin{tabular}{|l|l|l|l|}
\hline Variable & Group I & Group II & P value \\
\hline Age $(\mathrm{yrs})($ Mean \pm SD $)$ & $46.37 \pm 10.28$ & $50.2 \pm 12.01$ & 0.16 \\
\hline Gender(m/f)(n) & $21 / 14$ & $19 / 16$ & \\
\hline ASA II/III(n) & $17 / 18$ & $16 / 19$ & \\
\hline
\end{tabular}

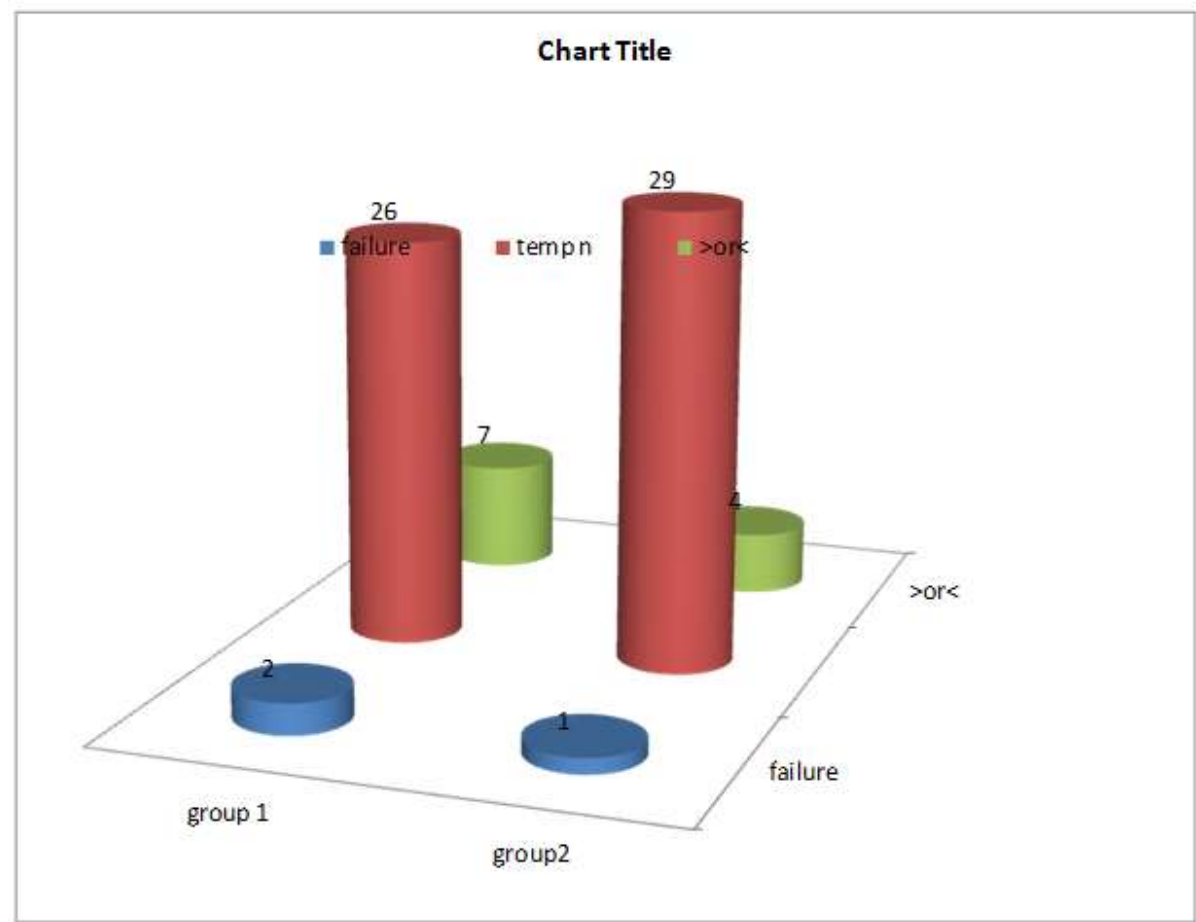

Fig 1.Temperature trends (chi square $=0.267 ; p=0.6$ )

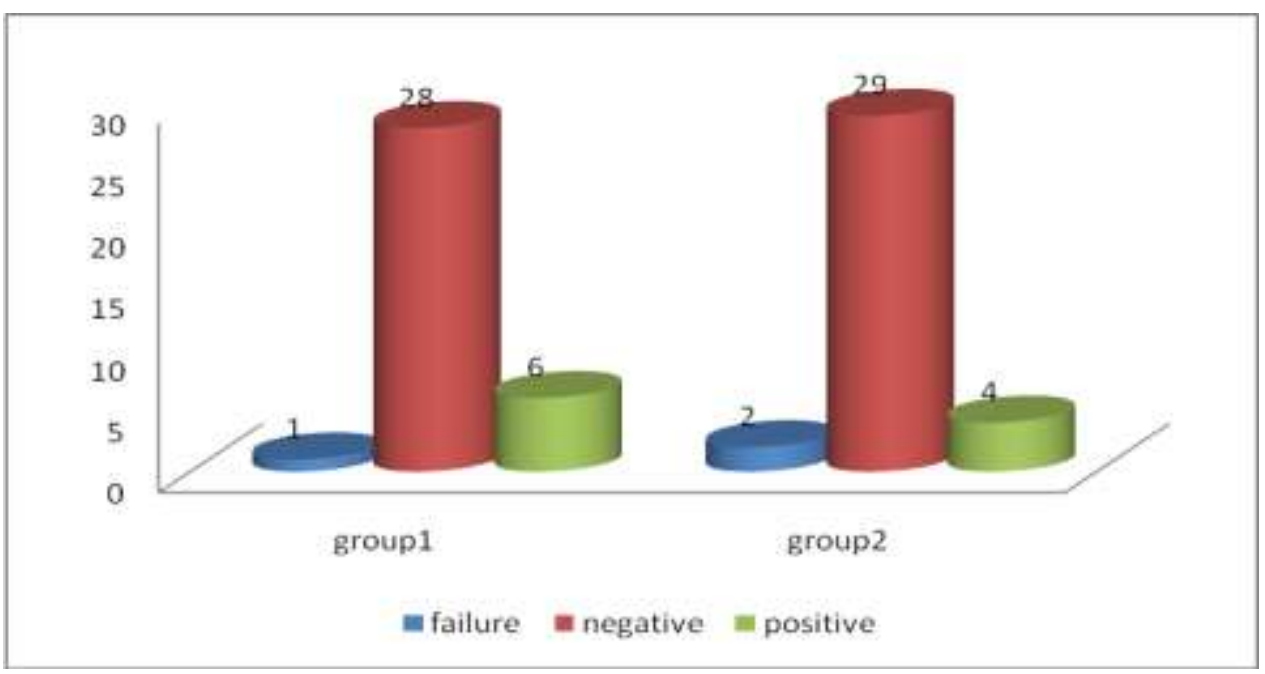

Fig.2 Endotracheal culture (chi square $=0.267 ; p=0.6$ ) 


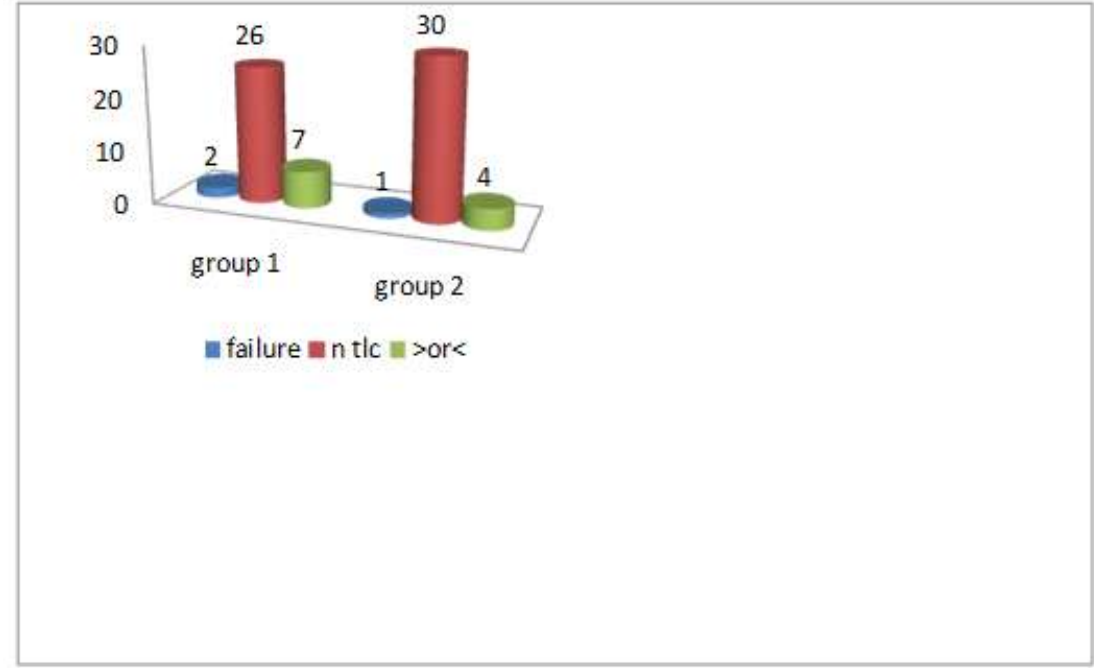

Fig3. Total leucocyte counts (chi square $=0.08 ; p=0.76$ )

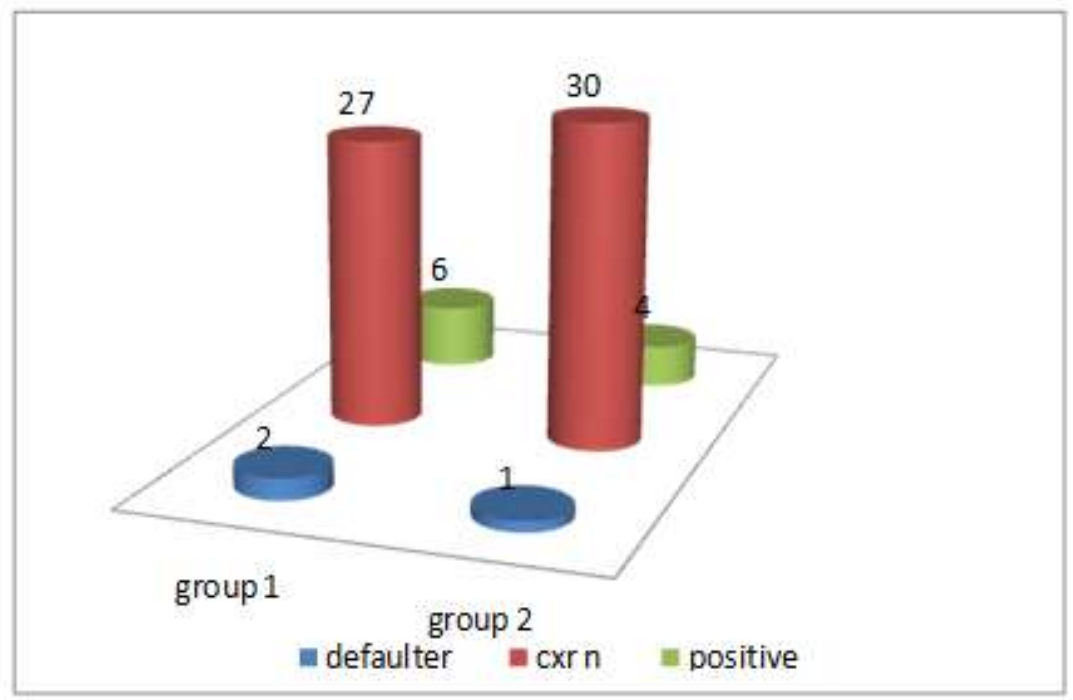

Fig4. Chest $\mathrm{X}$ ray (chi square $=0.06 ; p=0.8$ )

\section{References:}

[1] Safdar et.al Crit Care Med , 33 (10), 2005,2184-93.

[2] DE Craven, LM Kunches, V Kilinsky, DA Lichtenberg, BJ Make, WR McCabe, Risk factors for pneumonia and fatality in patients receiving continuous mechanical ventilation,.AmRevRespir Dis ,133(5), 1986,792-796.

[3] OC Tablan, LJ Anderson, R Besser, C Bridges, R Hajjeh, Healthcare Infection Control Practices Advisory Committee, Centers for Disease Control and Prevention, Guidelines for preventing health-careassociated pneumonia, 2003: recommendations of the CDC and the Healthcare Infection Control Practices Advisory Committee, MMWR RecommRep 53, 2004,1-36.

[4] J Chastre, JY Fagon,Ventilator-associated pneumonia,Am J RespirCrit Care Med,165(7), 2002,867-903.

[5] J Rello, DA Ollendorf, G Oster, V Montserrat, L Bellm, R Redman, MH Kollef,Epidemiology and outcomes of ventilator-associated pneumonia in a large US database,Chest ,122(6), 2002,2115-21.

[6] Robert A, Marc J M Bonten, Ventilator associated pneumonia: Preventing the inevitable, Oxford journal 52(1), 2011, 115-21.

[7] DJ Cook, SD Walter, RJ Cook, LE Griffith, GH Guyatt, D Leasa, RZ Jaeschke, C Brun-Buisson. "Incidence of and risk factors for ventilator-associated pneumonia in critically ill patients". Ann Intern Med, 129 (6), 1998, 433-40.

[8] JL Vincent, J Rello, J Marshall, Silva E, Anzueto A, Martin CD, Moreno R, J Lipman , C Gomersall , Y Sakr, K Reinhart ,International study of the prevalence and outcomes of infection in intensive care units, JAMA,302(21), 2009,2323-2329. 
[9] Safdar N, Crnich CJ, Maki DG. The pathogenesis of ventilator-associated pneumonia: its relevance to developing effective strategies for prevention. Respir Care,50(6),2005,725-739.

[10] LG Donowitz, MC Page, BL Mileur, SH Guenthner, Alteration of normal gastric flora in critical care patients receiving antacid and cimetidine therapy, Infect Control, 7(1),1986, 23-26.

[11] M Garrouste-Orgeas, S Chevret, G Arlet, O Marie, M Rouveau, N Popoff,Oropharyngeal or gastric colonization and nosocomial pneumonia in adult intensive care unit patients, A prospective study based ongenomic DNA analysis. Am J RespirCrit Care Med 156(5) ,1997,1647-1655. 